\title{
Magnetic phase transitions in the system $\mathrm{La}_{1-x} \mathrm{Bi}_{x} \mathrm{MnO}_{3+\lambda}$
}

\author{
I. O. Troyanchuk and O. S. Mantytskaja \\ Institute of Solid State and Semiconductor Physics of National Academy of Sciences of Belarus \\ 17 P. Brovki Str., Minsk 220072, Belarus \\ E-mail: troyan@ifttp.bas-net.by \\ H. Szymczak
}

Institute of Physics of Polish Academy of Sciences 32/46 Al. Lotnikow Str., Warsaw PL-02-668, Poland

\section{Yu. Shvedun}

B. Verkin Institute for Low Temperature Physics and Engineering of the National Academy of Science of Ukraine, 47 Lenin Ave., Kharkov 61103, Ukraine

Received January 4, 2002

The crystal structure and magnetic properties of the $\mathrm{La}_{1-x} \mathrm{Bi}_{x} \mathrm{MnO}_{3+\lambda}$ system $(0 \leq x \leq 1$; $\lambda \leq 0.08)$ are studied as functions of the oxygen and bismuth contents. In oxidized samples $\mathrm{La}_{1-x} \mathrm{Bi}_{x} \mathrm{MnO}_{3+\lambda}$ a phase transition from a ferromagnetic state (rhombohedric phase) to a state of the spin glass type (quasitetragonal phase) is observed with increase of the bismuth concentration. The reduced samples $\mathrm{La}_{1-x} \mathrm{Bi}_{x} \mathrm{MnO}_{3}$ are weak ferromagnets down to $x \leq 0.6$ and then transform into a ferromagnetic state. It is supposed that the $\mathrm{Bi}^{3+}$ ions stabilize the $d_{x^{2}-y^{2}}$ orbitals in the nearest $\mathrm{Mn}^{3+}$ ions whereas the $d_{z^{2}}$ orbitals of the $\mathrm{La}^{3+}$ ions are stabilized. The orbitally disordered phases and $d_{x^{2}-y^{2}}$ - orbitally ordered phases are ferromagnetic, the $d_{z^{2}}$ - orbitally ordered phases show antiferromagnetic ordering, and the state of the orbital glass type corresponds to a state of the spin glass type.

PACS: 72.15.Gd, 75.30.Kz, 75.70.Pa

\section{Introduction}

Recently considerable interest has been attracted to strongly correlated systems with perovskite-like structure. The variety of triple perovskites such as $\mathrm{RMO}_{3}$ ( $\mathrm{R}$ is a rare-earth metal, $\mathrm{M}$ is a transition metal) is quite great, but with variation of the rare-earth element $(\mathrm{R}=\mathrm{La}, \mathrm{Pr}, \mathrm{Nd}$ etc. $)$ and the addition of the practically unlimited set of solutions $\mathrm{R}_{1-x} \mathrm{~A}_{x} \mathrm{MnO}_{3}$, the number of combinations becomes extremely large.

Manganites $\mathrm{R}_{1-x} \mathrm{~A}_{x} \mathrm{MnO}_{3}$ have interesting and unusual properties. These compounds contain ions with orbital degeneracy or Jahn-Teller (JT) ions (in our case $\mathrm{Mn}^{3+}$ ) [1]. Thus their properties differ appreciably from those of the corresponding sub- stances with the «ordinary» ions: the crystal structure turn out to be distorted, structural phase transitions and transitions in a magnetic subsystem [2-6] are frequently observed in them, and in many cases they have anomalously strong magnetic anisotropy and magnetostriction $[7,8]$. Such phenomena are connected with an interaction of the JT ions and are called the cooperative Jahn-Teller effect (CJTE). Distortions of the crystal lattice are caused by the fact that ion $\mathrm{Mn}^{3+}$ is degenerate with respect to the $d$ orbitals: the crystal field splits the atomic $d$ level into two- and threefold degenerate sublevels $e_{g}$ and $t_{2 g}$ (the $e_{g}$ state is characterized by the real wave functions $d_{z^{2}}$ and $d_{x^{2}-y^{2}}$ ). The first of them lies above the second one, and consequently the four $d$ electrons of the $\mathrm{Mn}^{3+}$ ion occupy 
the level $t_{2 g}$ completely and the level $e_{g}$ only in part. This also makes CJTE possible, which reduces the energy of such a degenerate system by lowering its symmetry, which lifts the degeneracy of the electronic levels.

Despite the numerous works devoted to research on manganites, many problems remain subject to discussion. The unusual magnetic behavior of bismuth-containing manganites is one such problem. While the lanthanum-based manganites ( $\mathrm{Ln}-\mathrm{La}, \mathrm{Y}$, rare-earth ion) are antiferromagnetic [9], bismuth manganite is a ferromagnetic insulator $[10,11]$. Moreover, while substitution of the lanthanoid by an alkaline-earth metal leads to a transition from the antiferromagnetic to a ferromagnetic metallic state [12], a similar substitution for $\mathrm{BiMnO}_{3}$-based manganites destroys the ferromagnetic order [13-15].

The aim of the present work is to study phase transitions in a system of manganites where $\mathrm{La}^{3+}$ ions are replaced by $\mathrm{Bi}^{3+}$ ions. Formally upon such a substitution the valence of the manganese ions should not change.

\section{Experiment}

Samples of the $\mathrm{La}_{1-x} \mathrm{Bi}_{x} \mathrm{MnO}_{3+\lambda}$ system were obtained by three different methods: in air, under a high pressure, and in vacuum. The initial reagents were oxides of $\mathrm{La}_{2} \mathrm{O}_{3}, \mathrm{Bi}_{2} \mathrm{O}_{3}$ and $\mathrm{Mn}_{2} \mathrm{O}_{3}$, mixed in the desired proportion. The manganite of lanthanum was obtained at $T=1500{ }^{\circ} \mathrm{C}$ in air. Synthesis of Bi-containing samples was carried out in the temperature range of $900-1150{ }^{\circ} \mathrm{C}$ with a subsequent slow cooling $\left(100{ }^{\circ} \mathrm{C} / \mathrm{h}\right)$. The temperature of synthesis decreased with increasing bismuth content from $1150{ }^{\circ} \mathrm{C}(x=0.2)$ to $900{ }^{\circ} \mathrm{C}(x=0.7)$. According to $\mathrm{x}$-ray phase analysis, in a sample with $x=0.7$ there were traces of extraneous phases; therefore in these states the saturated concentration of bismuth is limited to a value of $65 \%$. A chemical analysis has shown that all compounds obtained in air have an excess of oxygen in comparison with the stoichiometric ratio. Substitution of the $\mathrm{La}^{3+}$ ions by $\mathrm{Bi}^{3+}$ has only a weak influence on the oxygen content. The chemical formula is $\mathrm{La}_{1-x} \mathrm{Bi}_{x} \mathrm{MnO}_{3.07 \pm 0,01}$. The samples prepared by the method described were reduced in quartz vacuum tubes at $900{ }^{\circ} \mathrm{C}$ in the presence of tantalum metal for absorption of allocated oxygen. Control of the oxygen maintenance was carried out by mass weighing of the sample before and after the reduction process. The chemical formula was «squared up» on loss of the mass. Compounds with $x \geq 0.7$ were obtained using a high-pressure technique (pressure $4 \mathrm{GPa}$, temperature $900{ }^{\circ} \mathrm{C}$, duration of synthesis $20 \mathrm{~min}$ ). These samples were characterized by the chemical composition $\mathrm{La}_{1-x} \mathrm{Bi}_{x} \mathrm{MnO}_{3 \pm 0,01}$.

$\mathrm{X}$-ray structure studies were carried out on a DRON-3 diffractometer in $\mathrm{Cr} K_{\alpha}$ radiation. Single-phase structures were selected for measurement of the magnetic and electric properties. Magnetic measurements were carried out on a commercial vibrating Foner magnetometer. The electroconductivity was measured by a standard four-probe technique. Indium contacts were used. They were applied using ultrasound.

\section{Results and discussion}

According to the $\mathrm{x}$-ray data, the samples of the $\mathrm{La}_{1-x} \mathrm{Bi}_{x} \mathrm{MnO}_{3.07}$ series obtained in air were characterized by rhombohedral distortions of the unit cell up to a concentration $x=0.4$. In samples with $x \geq 0.5$ the type of distortions changed to tetragonal. The compounds reduced in vacuum and obtained under a high pressure had a monoclinic or orthorhombic deformed unit cell.

Upon substitution of lanthanum by bismuth, the volume of the unit cell varied only slightly, apparently because of the similarity of the ionic radii of

Table 1

Unit cell parameters of $\mathrm{La}_{1-x} \mathrm{Bi}_{x} \mathrm{MnO}_{3,07}$ compounds obtained in air

\begin{tabular}{c|c|c|c|c|c}
\hline \hline Composition & Symmetry & $a, \AA$ & $c, \AA$ & $\alpha$, deg & $V, \AA^{3}$ \\
\hline \hline $\mathrm{LaMnO}_{3.07}$ & $\mathrm{R}$ & 7.8105 & - & 90.46 & 59.55 \\
$\mathrm{La}_{0.8} \mathrm{Bi}_{0.2} \mathrm{MnO}_{3.07}$ & $\mathrm{R}$ & 7.8165 & - & 90.45 & 59.69 \\
$\mathrm{La}_{0.7} \mathrm{Bi}_{0.3} \mathrm{MnO}_{3.07}$ & $\mathrm{R}$ & 7.8191 & - & 90.34 & 59.75 \\
$\mathrm{La}_{0.6} \mathrm{Bi}_{0.4} \mathrm{MnO}_{3.07}$ & $\mathrm{R}$ & 7.8198 & - & 90.27 & 59.7 \\
$\mathrm{La}_{0.5} \mathrm{Bi}_{0.5} \mathrm{MnO}_{3.07}$ & $\mathrm{~T}$ & 3.9098 & 3.9352 & - & 60.156 \\
$\mathrm{La}_{0.4} \mathrm{Bi}_{0.6} \mathrm{MnO}_{3.07}$ & $\mathrm{~T}$ & 3.9048 & 3.9409 & - & 60.09 \\
\hline \hline
\end{tabular}




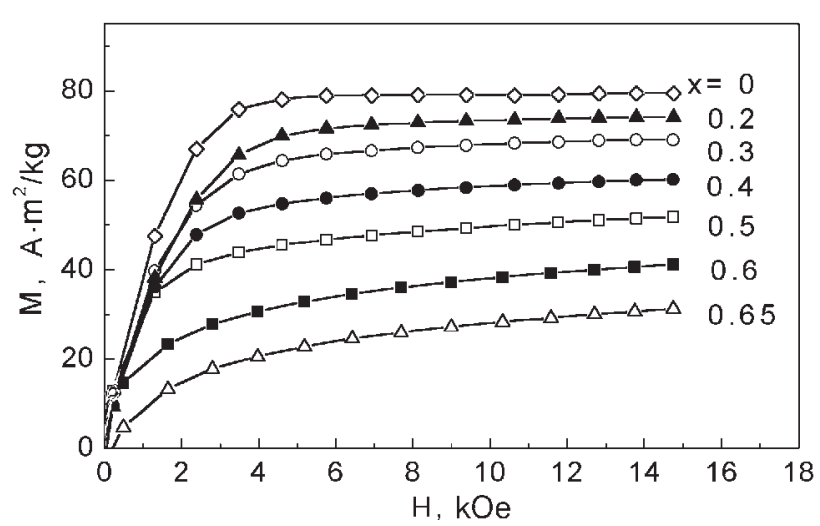

Fig. 1. Magnetic field dependence of the magnetization in the $\mathrm{La}_{1-x} \mathrm{Bi}_{x} \mathrm{MnO}_{3.07}$ system.

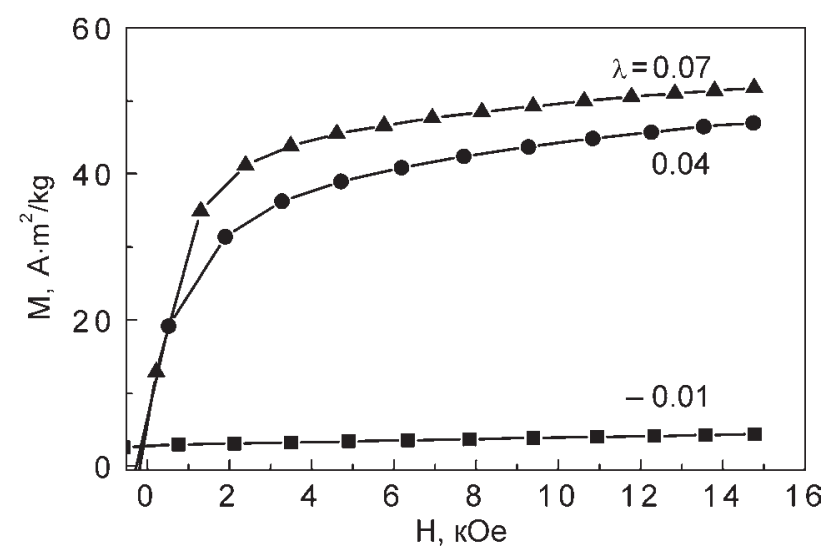

Fig. 3. Magnetic field dependence of the magnetization in the $\mathrm{La}_{0.5} \mathrm{Bi}_{0.5} \mathrm{MnO}_{3+\lambda}$ system.

Table 2

Unit cell parameters of $\mathrm{La}_{1-x} \mathrm{Bi}_{x} \mathrm{MnO}_{3+\lambda}$ compounds obtained in vacuum

\begin{tabular}{c|c|c|c|c|c}
\hline \hline Composition & Symmetry & $a, \AA$ & $b, \AA$ & $c, \AA$ & $V, \AA^{3}$ \\
\hline \hline $\mathrm{La}_{0.5} \mathrm{Bi}_{0.5} \mathrm{MnO}_{3.07}$ & $\mathrm{~T}$ & 3.9098 & 3.9352 & - & 60.15 \\
$\mathrm{La}_{0.5} \mathrm{Bi}_{0.5} \mathrm{MnO}_{3.04}$ & $\mathrm{O}$ & 5.5456 & 5.5879 & 7.7862 & 61.095 \\
$\mathrm{La}_{0.5} \mathrm{Bi}_{0.5} \mathrm{MnO}_{2.99}$ & $\mathrm{O}^{\prime}$ & 5.501 & 5.8266 & 7.6635 & 61.41 \\
\hline \hline
\end{tabular}

$\mathrm{La}^{3+}$ and $\mathrm{Bi}^{3+}$. The unit cell parameters of some solid solutions are given in Tables 1 and 2. According to structural studies [16], the excess over stoichiometric oxygen in the system $\mathrm{LaMnO}_{3+\lambda}$ is a consequence of the formation of an equivalent number of vacancies of $\mathrm{La}^{3+}$ and $\mathrm{Mn}^{3+}$ ions. Apparently this mechanism of nonstoichiometry is pertinent to system $\mathrm{La}_{1-x} \mathrm{Bi}_{x} \mathrm{MnO}_{3+\lambda}$ also, as the oxygen ions cannot occupy interstitial positions in the closepacked perovskite structure.

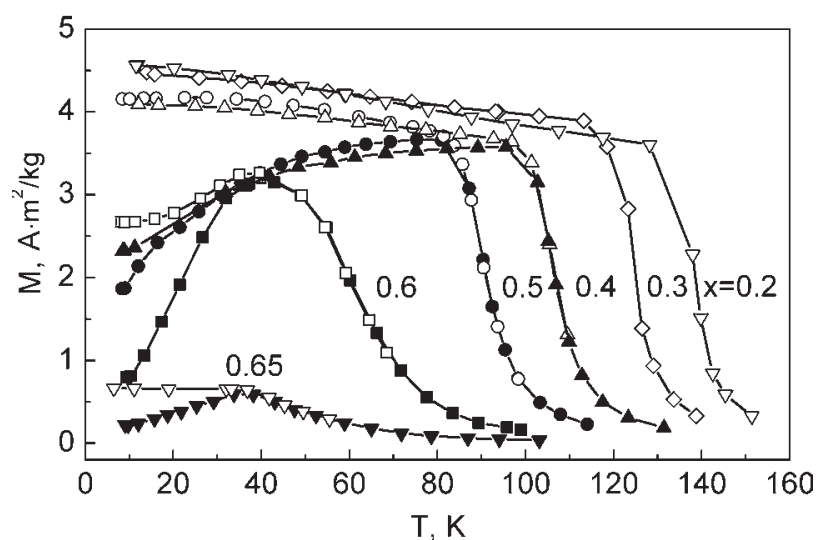

Fig. 2. Temperature dependence of the magnetization in the $\mathrm{La}_{1-x} \mathrm{Bi}_{x} \mathrm{MnO}_{3.07}$ system (ZFC curve - filled symbols, FC - unfilled symbols).
Field curves of the magnetization $M(H)$ at liquid helium temperature for several samples prepared in air are shown in Fig. 1. $\mathrm{LaMnO}_{3,07}$ is ferromagnetic, as the specific magnetization corresponds to a parallel orientation of the magnetic moments of the manganese ions. With increase of the bismuth content up to $x=0.65$, a gradual decrease of the spontaneous magnetization was observed. In compounds with $x=0.6$ and $x=0.65$ the magnetization remains unsaturated in fields up to $16 \mathrm{kOe}$, as is typical for

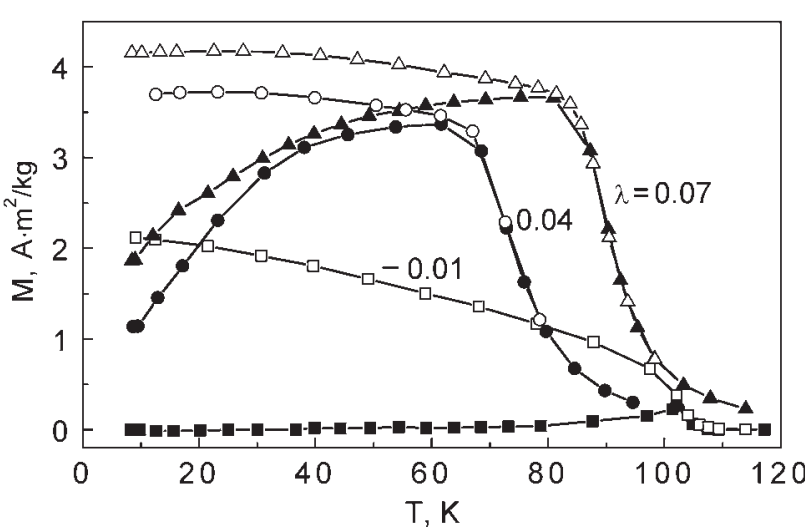

Fig. 4. Temperature dependence of the magnetization in the $\mathrm{La}_{0.5} \mathrm{Bi}_{0.5} \mathrm{MnO}_{3+\lambda}$ system (ZFC curve - filled symbols, FC - unfilled symbols). 
magnets with weak cooperative exchange interactions. Ferromagnetic solutions $(x \leq 0.4)$ are soft magnetic materials whereas in samples with $x \leq 0.5$ the coercive force sharply increases. The temperature curves of the magnetization obtained in a rather small external magnetic field of 100 Oe are shown in Fig. 2. The measurements were performed in a mode of heating after cooling in a field (FC) and without a field (ZFC). It follows from the diagrams that the Curie temperature decreases with increase of bismuth content. The ZFC magnetization of a sample with $x=0.6$ has a wide maximum, which is typical for a magnet near the concentration transition from a ferromagnetic state to the state such as a spin glass. On further increase of the bismuth content the magnetic susceptibility sharply decreases. The ZFC magnetization in a sample with $x=0.65$ has a well-defined maximum. Near the temperature of the maximum the $\mathrm{ZFC}$ and $\mathrm{FC}$ magnetizations diverge. Taking into account the form of $M(H)$ curve we believe that in solutions with $x=0.65$ the long-range magnetic order is apparently destroyed.

The compound $\mathrm{La}_{0.5} \mathrm{Bi}_{0.5} \mathrm{MnO}_{3.07}$ was reduced under various conditions. As a result, two compounds $-\mathrm{La}_{0.5} \mathrm{Bi}_{0.5} \mathrm{MnO}_{3.04}$ and $\mathrm{La}_{0.5} \mathrm{Bi}_{0.5} \mathrm{MnO}_{2.99}$ are obtained. The partially reduced sample had an O-orthorhombically distorted unit cell $(a<c \sqrt{2}<b)$, whereas the strongly reduced had $\mathrm{O}^{\prime}$-orthorhombically deformed unit cell $(c \sqrt{2}<a<b)$. It should be noted, that $\mathrm{O}^{\prime}$-orthorhombic distortions in manganites indicate orbital ordering [17]. The field curves of the magnetization at liquid helium temperature for reduced samples are plotted in Fig. 3. The magnetizations of compounds with $\lambda=0.04$ and $\lambda=0.07$ differ slightly, whereas the composition with $\lambda=-0.01$ has a very small spontaneous magnetization (about $0.2 \mu_{B}$ in formula units).

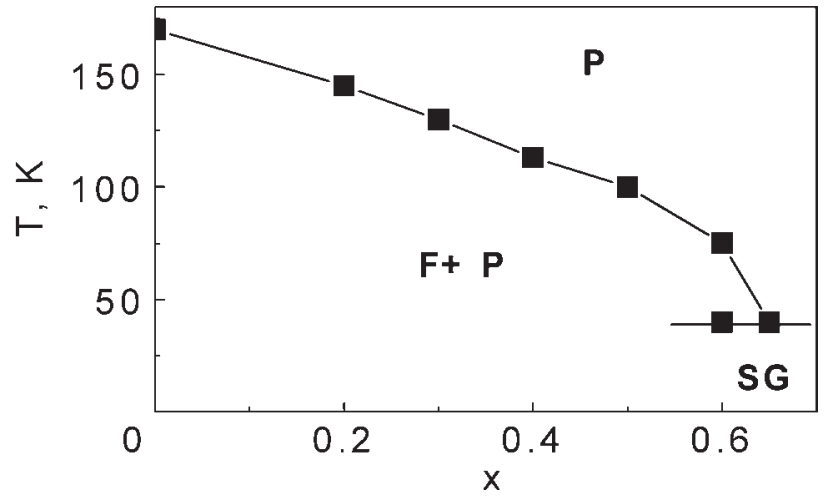

Fig. 5. Magnetic phase diagram of the $\mathrm{La}_{1-x} \mathrm{Bi}_{x} \mathrm{MnO}_{3.07}$ system. P - paramagnetic state, $\mathrm{F}$ - ferromagnetic, $\mathrm{SG}-$ spin glass.

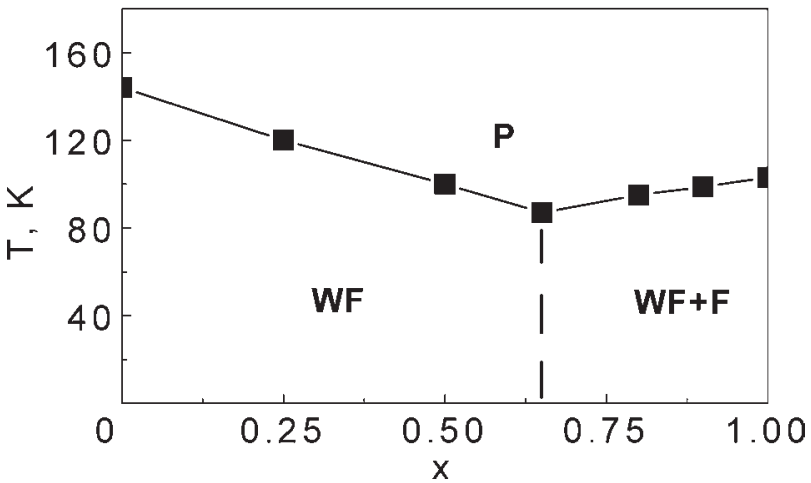

Fig. 6. Magnetic phase diagram of the $\mathrm{La}_{1-x} \mathrm{Bi}_{x} \mathrm{MnO}_{3}$ system. WF - weak ferromagnetic state, $\mathrm{P}-$ paramagnetic, $\mathrm{F}$ - ferromagnetic.

With loss of oxygen the critical temperature at which a spontaneous magnetization appears at first decreased and then increased again and, moreover, the transition to the paramagnetic state became sharper (Fig. 4).

It should be noted that with decrease of the oxygen content in the reduced manganites the magnetic anisotropy increased sharply. Compounds with stoichiometric oxygen $(x \leq 0.65)$ are hard, strongly anisotropic magnets. The coercive force $H_{C}$ at helium temperature reaches $15 \mathrm{kOe}$, whereas for the ferromagnetic oxidized compositions $H_{C}=100$ Oe.

As compounds with bismuth content above $65 \%$ may not be obtained in air, synthesis of solutions in the range $0.7 \leq x \leq 1$ is performed at high pressures. We have found out that the Curie temperature increases smoothly with increase of bismuth content from $87 \mathrm{~K}(x=0.65)$ up to $108 \mathrm{~K}$ for $\mathrm{BiMnO}_{3}$. The spontaneous magnetization grows sharply on going from a solution with $x=0.8$ to a solution with $x=0.9$. It is necessary to note that the growth of the spontaneous magnetization correlates with a change of the unit cell symmetry type from orthorhombic $(x=0.8)$ to monoclinic $(x=0.9)$.

Measurements of the electrical conductivity are carried out in a temperature range of 77-350 K. All of the compounds under study had a semiconductor character of the temperature dependence of the electrical resistance. Near the temperature of magnetic ordering for structures with $x \leq 0.4$ a maximum of the magnetoresistance was observed (up to $40 \%$ in a field $9 \mathrm{kOe}$ ), but magnetic ordering did not change the type of the conductivity.

Figure 5 presents the magnetic phase diagram of the $\mathrm{La}_{1-x} \mathrm{Bi}_{x} \mathrm{MnO}_{3,07}$ system with an excess of oxygen. The ferromagnetic state is gradually destroyed with increase of the bismuth content, until in the 
solution with $x=0.65$ the state of the cluster-type spin glass is not stabilized.

The magnetic phase diagram of the stoichiometric structures $\mathrm{La}_{1-x} \mathrm{Bi}_{x} \mathrm{MnO}_{3}$ is shown in Fig. 6 . $\mathrm{LnMnO}_{3}$ is a weak ferromagnet with Néel temperature $T_{N}=144 \mathrm{~K}$. With increase of the bismuth content up to $x=0.65$ the stoichiometric structures still show the properties inherent to a weak ferromagnet. The Néel temperature gradually decreases to $87 \mathrm{~K}(x=0.65)$, apparently is because of a decrease of the $\mathrm{Mn}-\mathrm{O}-\mathrm{Mn}$ bond angles. The lower the $\mathrm{Mn}-\mathrm{O}-\mathrm{Mn}$ angle, the lower are the width of the $3 d$ band and the temperature of magnetic ordering [18-21]. It is well known that in $\mathrm{LaMnO}_{3}$ antiferrodistortion ordering of the $d_{z^{2}}$ orbitals occurs in the $a b$ plane as the exchange interaction becomes ferromagnetic in this plane and antiferromagnetic along the $c$ axis [18]. The magnetic moments are oriented along the $b$ axis. In Bi-substituted stoichiometric manganites $(x \sim 0.6)$ another type of antiferromagnetic and orbital ordering is in principle possible. However, in order to ascertain the features of the magnetic and structural state, neutron diffraction studies are required. In bismuth manganite the orbital state $d_{x^{2}-y^{2}}$ is stabilized, leading to an isotropic ferromagnetic state [14,21]. $\mathrm{BiMnO}_{3}$ is a soft magnetic material, despite the orbital ordering $[14,22]$. In solutions with a high bismuth content the magnetic state is most likely a two-phase one. The sample consists of ferromagnetic and antiferromagnetic areas. We believe that each type of magnetic state is characterized by its own type of orbital state.

It is well known that in manganites the substitution of the rare-earth ions $\mathrm{R}^{3+}$ and $\mathrm{La}^{3+}$ by an alkaline-earth ion results in orbital disordering and stabilization of the ferromagnetic state. This process is caused by two factors. First, vacancies appear in the orbitally ordered lattice (not Jahn-Teller ions $\mathrm{Mn}^{4+}$ ), and that destabilizes a cooperative orbital ordering. Second, distortions of the crystal lattice decrease due to optimization of the cation's sizes, whereas the $\mathrm{Mn}-\mathrm{O}-\mathrm{Mn}$ bond angle which describes the width of the $3 d$ band, is increased. Under these conditions the static Jahn-Teller distortions transform to dynamic ones, and the exchange interactions of the $\mathrm{Mn}^{3+}-\mathrm{O}-\mathrm{Mn}^{3+}$ and $\mathrm{Mn}^{3+}-\mathrm{O}-\mathrm{Mn}^{4+}$ types become ferromagnetic [18]. The establishment of magnetic order in structures with a large enough width of the $3 d$ band leads to transition in metallic state due to overlapping of the bands formed mainly by $3 d$ and $2 p$ states.
In manganites containing a large quantity of $\mathrm{Bi}^{3+}$ ions the appearance of tetravalent ions of manganese does not induce ferromagnetism (Fig. 3). The presence of $\mathrm{Mn}^{4+}$ ions promotes the destruction of cooperative orbital ordering and the occurrence of properties inherent to spin glasses. Thus the compounds remain insulators. We believe that in compounds with a large content of $\mathrm{Bi}^{3+}$ ions the local static Jahn-Teller distortions are not removed. This is explained by the tendency of $\mathrm{Bi}^{3+}$ ions to form strongly anisotropic covalent $p s$ bonds. These bonds promote local crystal structure distortions, which is the reason why the local Jahn-Teller orbital ordering is preserved. Indeed, the temperature of the charge and orbital ordering in $\mathrm{Bi}_{0.5} \mathrm{Sr}_{0.5} \mathrm{MnO}_{3}$ is unusually high, above $500 \mathrm{~K}$ $[23,24]$, whereas the characteristic temperature of charge and orbital ordering in the structures $\mathrm{R}_{0.5} \mathrm{Sr}_{0.5} \mathrm{MnO}_{3}$ is $150 \mathrm{~K}[25,26]$. Due to the local static Jahn-Teller distortions, the anisotropic character of the exchange interactions between the manganese ions persist. On the other hand, in these samples, due to fluctuations of the composition, microareas may exist in which the static Jahn-Teller distortions are removed. These microareas are ferromagnetic. The competition between ferromagnetism and antiferromagnetism results in a spin glass state of a cluster type. It should be noted that orbital and charge ordering in manganites are transitions of the martensitic type [27,28]. Therefore orbitally two-phase states in manganites should be realized at the concentration and temperature boundaries of the orbital order-disorder transitions.

\section{Summary}

The crystal structure and magnetic properties of both oxidized $\mathrm{La}_{1-x} \mathrm{Bi}_{x} \mathrm{MnO}_{3.07}$ and reduced $\mathrm{La}_{1-x} \mathrm{Bi}_{x} \mathrm{MnO}_{3}$ perovskites have been studied. It is shown that oxidized $\mathrm{La}_{1-x} \mathrm{Bi}_{x} \mathrm{MnO}_{3.07}$ series exhibit a concentration transition from ferromagnetic state to spin glass at $x \geq 0.65$ whereas reduced $\mathrm{La}_{1-x} \mathrm{Bi}_{x} \mathrm{MnO}_{3}$ perovskites transform from weak ferrimagnet state to ferromagnetic one at $x>0.7$ through a mixed magnetic state. The results obtained are discussed in terms of interplay between orbital ordering and type of magnetic ground state.

This work was supported in part by the Belarus Fund of Basic Research (Project F00-223). 
1. K. I. Kugel and D. I. Khomskii, Usp. Fiz. Nauk 136, 621 (1982) [Sov. Phis. Usp. 25, 231 (1982)].

2. V. Eremenko, S. Gnatchenko, N. Makedonska, Yu. Shabakayeva, M. Shvedun, V. Sirenko, J. FinkFinowicki, K. Kamenev, G. Balakrishnan, and P. Mck Paul, Fiz. Nizk. Temp. 27, 1258 (2001) [Low Temp. Phys. 27, 930 (2001)].

3. R. Mahendiran, M. R. Ibarra, F. Maigan, A. Arulraj, R. Mahesh, O. Revcau, and C. N. R. Rao, Phys. Rev. Lett. 82, 2191 (1999).

4. P. M. Woodward, D. E. Cox, T. Vogt, C. N. Rao, and A. K. Cheetham, Chem. Mater. 11, 3528 (1999).

5. I. O. Shklyarevskiy, M. Yu. Shvedun, S. L. Gnatchenko, P. J. M. van Bentum, P. C. M. Christianen, J. C. Maan, K. V. Kamenev, G. Balakrishnan, P. Mck Paul, and J. Fink-Finowicki, Fiz. Nizk. Temp. 27, 1250 (2001) [Low Temp. Phys. 27, 923 (2001)]

6. I. O. Troyanchuk, D. A. Efimov, V. V. Eremenko, V. A. Sirenko, H. Szymszak, and A. Nabialek, Fiz. Nizk. Temp. 26, 39 (2000) [Low. Temp. Phys. 26, 28 (2000)].

7. V. V. Eremenko, V. A. Sirenko, H. Szymszak, and A. Nabialek, Fiz. Nizk Temp. 25, 311 (1999) [Low Temp. Phys. 25, 221 (1999)].

8. V. M. Loktev and Yu. G. Pogorelov, Fiz. Nizk. Temp. 26, 231 (2000) [Low Temp. Phys. 26, 171 (2000)].

9. M. R. Ibarra, P. A. Alagarabel, C. Marguina, J. Blasco, and J. Garsia, Phys. Rev. Lett. 75, 3541 (1995)

10. S. Quezel-Ambrunaz, Bull. Soc. Fr. Mineral et Crystallogr. 91, 339 (1968)

11. F. Sugawara, S. Iida, Y. Syono, and S. Akimoto, J. Phys. Soc. Jpn. 20, 1529 (1965).

12. V. A. Bokov, N. A. Milnikova, S. A. Kizhaev, M. F. Brizhina, and I. A. Grigoryan. Fiz. Tverd. Tela 7, 3695 (1965) (in Russian).
13. G. H. Jonker and J. H. van Santen, Physica 16, 337 (1950).

14. I. O. Troyanchuk, N. V. Kasper, O. S. Mantytskaya, and S. N. Pastushonok, Sov. Phys. JETP 78, 212 (1994).

15. I. O. Troyanchuk, N. V. Samsonenko, E. F. Shapovalova, I. M. Kolesova, and H. Szymczak, J. Phys.: Condens. Matter. 8, 11205 (1996).

16. H. Chiba, T. Atou, and Y. Syono, J. Solidi Status Chem. 132, 139 (1997).

17. J. A. M. van Roosmalen and E. H. P. Cordfunke, J. Solid. State Chem. 110, 109 (1994).

18. D. Gudenaf, Magnetizm i himicheskaya soyaz, Metalurgiya, Moskva (1968) (in Russian).

19. P. G. De Gennes, Phys. Rev. 18, 1, 141 (1960).

20. E. E. Havinga, Philips Res. Rep. 21, 432 (1966).

21. T. Atou, H. Chiba, K. Ohoyama, Y. Yamagnuchi, and Y. Syono, J. Solid. State Chem. 145, 639 (1999).

22. I. O. Troyanchuk and V. N. Derkachenko, Fiz. Tverd. Tela 32, 2474 (1990) (in Russian).

23. N. V. Kasper, I. O. Troyanchuk, A. N. Chobot, H. Szymczak, and J. Fink-Finowicki, J. Phys.: Condens. Matter. 9, 7455 (1997).

24. J. L. Carcia-Munos, C. Froutera, M. A. G. Aranda, A. Liobet, and C. Ritter, Phys. Rev. B63, 064415 (2001).

25. F. Millange, S. de Brion, and G. Chouteau, Phys. Rev. B62, 9, 5619 (2000).

26. H. Kuwahara, Y. Tomioka, A. Asamitsu, Y. Moritomo, and Y. Tokura, Science 270, 961 (1995).

27. V. Podzorov, B. G. Kim, V. Kiryukhin, M. E. Gershenson, and S-W. Cheong, Phys. Rev. B64, 140406 (2001)

28. I. O. Troyanchuk, A. I. Akimov, L. A. Bliznjuk, and N. V. Kasper, J. Alloys and Compounds 228, 83 (1995). 